\title{
Serum Oxidative-Antioxidative Status in Patients With Alkaptonuria
}

\author{
Eman M. Albatayneha, f, Mohammed S. Al-Sbou ${ }^{\mathrm{b}}$, Samir S. Mahgoub ${ }^{\mathrm{c}}$, \\ Nesrin R. Mwafi ${ }^{\mathrm{d}}$, Nedal A. Alnawaiseh ${ }^{\mathrm{e}}$
}

\begin{abstract}
Background: Alkaptonuria (AKU) is a rare genetic disease associated with the deposition of melanin-like pigments (ochronosis) in connective tissues. However, data regarding the effect of oxidative stress products on disease pathogenesis are limited. The purpose of this study was to investigate oxidative stress and related factors in patients with alkaptonuria and compare the findings with those in healthy control subjects.
\end{abstract}

Methods: The study sample comprised of 21 AKU patients and 19 age- and sex-matched healthy controls. Serum samples were obtained to detect the total antioxidative capacity (TAC), and oxidation degradation products of thiobarbituric acid-reactive substances, protein carbonyls, advanced oxidation protein products, and homogentisic acid levels in urine were determined.

Results: Serum TAC, oxidation degradation products of thiobarbituric acid-reactive substances, and protein carbonyl levels in the AKU group were higher than those measured for the control subjects, and the difference was statistically significant $(\mathrm{P}<0.05)$. Moreover, a positive correlation was found between the patient's serum protein carbonyl, patient's age and AKU severity score $(r=0.492$ and 0.746 , respectively; $\mathrm{P}<0.05)$. Furthermore, the protein carbonyl serum levels can be used to predict the disease severity score in alkaptonuria patients $(\mathrm{P}<0.05)$.

Conclusions: In sum, the study results provide further support for the role of oxidation in the pathogenesis of alkaptonuria, suggesting pres-

Manuscript submitted February 27, 2019, accepted March 19, 2019

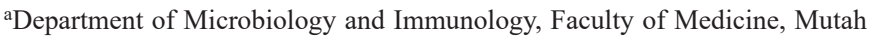
University, Mutah, Jordan

${ }^{b}$ Department of Pharmacology, Alkaptonuria Research Office, Faculty of Medicine, Mutah University, Mutah, Jordan

cDepartment of Biochemistry and Molecular Biology, Faculty of Medicine, Al-Minia University, Al-Minia, Egypt

${ }^{\mathrm{d} D e p a r t m e n t}$ of Biochemistry and Molecular Biology, Alkaptonuria Research Office, Faculty of Medicine, Mutah University, Mutah, Jordan

eDepartment of Public Health, Faculty of Medicine, Mutah University, Mutah, Jordan

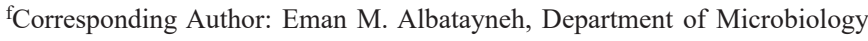
and Immunology, Faculty of Medicine, Mutah University, Mutah, Jordan. Email: emanbatayneh@yahoo.com

doi: https://doi.org/10.14740/jocmr3801 ence of a more complex relationship than what has been previously assumed. Thus, further studies are needed to clarify these conflicting results.

Keywords: Oxidative stress; Alkaptonuria; Protein carbonyl; Total antioxidative capacity; Oxidation degradation products of thiobarbituric acid-reactive substances; Advanced oxidation protein products

\section{Introduction}

Alkaptonuria (AKU) is a highly rare inborn metabolic error associated with a defective catabolism of phenylalanine and tyrosine, leading to increased systemic levels of homogentisic acid (HGA), which is prone to oxidation/polymerization reactions, causing production of a melanin-like pigment. Once the pigment is deposited within connective tissues (mainly in joints, spine, and cardiac valves), a classical bluish-brown discoloration is imparted, leading to a phenomenon known as "ochronosis", which is the hallmark of AKU. The clinical features of AKU are characterized by homogentisic aciduria, bluish-black discoloration of connective tissues, and arthropathy of weight-bearing joints such as hips and knees, and spondyloarthropathy [1]. Complications that are commonly associated with this disease include stone formation in kidneys, prostate, gall bladder and salivary glands, rupture of tendons, ligaments, and muscles, and cardiovascular manifestations (cardiac arrhythmias, aortic valve disease) [2].

Thus, there is an urgent need to identify biomarkers for monitoring AKU progression and evaluating response to treatment. Studies on the molecular mechanisms for the effect of the ochronotic pigment in inducing inflammation in AKU are relatively recent and scarce, and are based on a range of in vitro and ex vivo human models [3]. Their findings indicate that, although AKU is a multisystemic disease, osteoarticular system is the most severely affected, and articular cartilage is the most highly damaged tissue. In pertinent research, ultrastructural observations were complemented with biochemical and proteomic characterization of chondrocytes isolated from the ochronotic cartilage of AKU patients, indicating that AKU chondrocytes are characterized by HGA-induced nitric oxide release, and oxidative stress [4-8]. This association was demonstrated in a number of studies. For example, it is assumed that lipid peroxidation is initiated by free-radical attack of 
chondrocyte membrane lipids, generating large amounts of reactive products [9]. Moreover, in "black" AKU chondrocytes, chondrocytes isolated from the ochronotic cartilage of AKU patients showed an increased post-translational oxidation of proteins [5]. Furthermore, findings reported in one study revealed interesting similarities with those obtained in proteomic studies on other rheumatic diseases, such as osteoarthritis, supporting the existence of oxidative stress and inflammation in AKU $[9,10]$. Moreover, the efficacy of antioxidant treatment in counteracting the production of HGA-induced melanin-like pigments and protein oxidation induced by HGA and its metabolites has also been demonstrated $[11,12]$.

Oxidative stress occurs due to an increase in the production of oxidants and/or a decrease in protective antioxidants. Oxygen is an essential element of aerobic life and oxidative metabolism, and represents a principal source of energy. However, when partially reduced, it generates reactive oxygen species (ROS). Hence, imbalance between radical and non-radical ROS can damage joint tissues by a variety of mechanisms, including peroxidation of lipid membranes and protein oxidation.

These ROS are free radicals containing one or more unpaired electrons, which can damage a wide variety of biomolecules and cell structures, with a half-life of only seconds. Therefore, in vivo determination of ROS is generally unfeasible. In contrast, having lifetimes ranging from hours to weeks, lipids, proteins, carbohydrates, and DNA after being modified by oxy-radicals can be measured with biochemical assays, which makes them ideal markers of oxidative stress. Many biomarkers have been developed to evaluate oxidative stress. The biomarker that is generally used to estimate protein oxidation is protein carbonyl (PC), which is derived from amino acids during metal-catalyzed oxidation of proteins in vitro and in vivo, thus representing a direct measure of the oxidative injury to these molecules [13]. Advanced oxidation protein products (AOPP), as a new marker of protein oxidation, have begun to attract the attention of various investigators. They are formed during oxidative stress by the action of chlorinated oxidants, mainly hypochlorous acid and chloramines, which are produced by myeloperoxidase in activated neutrophils. Given the above, AOPP has the potential to serve as a marker of oxidative stress specific to proteins in mammalian systems [10]. Lipid peroxidation is initiated when free radicals interact with polyunsaturated fatty acids. For instance, in cell membranes, this may result in a chain reaction forming lipid hydroperoxides. Analysis of thiobarbituric acid-reactive substances (TBARS) in plasma is a widely used method for estimating the degree of lipid peroxidation. All aforementioned markers have been identified in oxidative stress generated in AKU $[5,9,14]$

To protect against toxic effects of oxidative products, the cell has developed antioxidant defense systems. Several enzymatic antioxidants exist in human body, such as superoxide dismutase (SOD); glutathione peroxidase/reductase system (GPX) and catalase (CAT); as well as non-enzymatic antioxidants, such as vitamin $\mathrm{C}, \mathrm{E}, \mathrm{Zn}, \mathrm{Cu}$ and uric acid. They are consequently referred to as total antioxidant capacity (TAC). The TAC concept emerged by considering the synergistic role of those antioxidants, rather than the simple sum of individual antioxidant actions [15].

An imbalance between pro-oxidative and anti-oxidant capacity has been postulated to be a crucial feature in the pathogenesis of inflammatory joint diseases, among which rheumatoid arthritis (RA) remains the most extensively studied [16-18]. In extant studies, RA patients showed a marked increase in ROS formation, lipid peroxidation, protein oxidation, and DNA damage, along with a decreased activity of antioxidant defense system. The resulting oxidative stress may contribute to tissue damage and hence to the chronicity of the disease [19]. However, research focusing specifically on patients with ankylosing spondylitis (AS) is limited, even though an increase in oxidative metabolism of the phagocyte system is commonly noted in this population.

Some authors have also reported that oxidative stress is more pronounced in the active disease status [20]. In extant literature, the scoring system for the alkaptonuria disease such as AKU Severity Score Index (AKUSSI) was assessed and correlated with various oxidative stress molecules. AKUSSI was initially developed as an assessment tool for determining disease severity. It can thus be used to compare disease severity between AKU patients and to measure the disease progression $[21,22]$.

The present study marks the first attempt to test the balance between serum antioxidant and the products of oxidative stress in patients with AKU and to compare these findings with those pertaining to age- and sex-matched healthy controls. Additionally, this is the first study in which the association of oxidation markers levels with the disease severity score, age and HGA serum levels is examined.

\section{Materials and Methods}

\section{Patients and sample collection}

The study sample included 21 AKU patients (mean age $37.9 \pm$ 12.6 years, range 13 - 58, 17 male, 4 female) and 19 age- and sex-matched healthy controls (mean age $36 \pm 10.6$ years, range 13 - 53, 13 male, 6 female). The AKU patients were registered in the Jordanian Society of Alkaptonuria and were diagnosed based on the results of laboratory investigations and clinical assessments. The AKU diagnosis was determined by observing the changes in urine color to a dark-black and via confirmation by quantitative measurements of urinary HGA levels using gas chromatography mass spectrophotometry (GCMS). Clinical examination was also performed to assess the disease signs and symptoms. Finally, radiological examination using X-ray of the joints was performed to evaluate the ochronotic arthropathy. The study protocol was approved by the Ethics Committee of the Mutah University. A full and very detailed medical history of all potential study participants had been taken prior to commencing the data collection. The aim was to exclude all individuals that suffer from any other chronic diseases or are undergoing any medical treatments. The AKU Severity Score questionnaires were completed by a physician, in line with the clinical examination and radiological X-ray findings and patients' complaints. 
Urine samples were collected during a 24-h period from 13 AKU patients and homogentisic acid was analyzed. After liquid-liquid extraction, the analyte was determined by the GCMS method following a 12-h fasting period. Venous blood samples $(20 \mathrm{~mL})$ were collected in the morning from all study participants, using vials containing sodium citrate as anticoagulant, and were immediately separated by centrifugation at 3,000 rpm for $10 \mathrm{~min}$ at $+4{ }^{\circ} \mathrm{C}$. The plasma samples were frozen at $-80{ }^{\circ} \mathrm{C}$ until AOPPs and MDA assays. Assays were carried out in duplicates.

Urinary homogentisic acid level measurement using gas chromatography and mass spectrometry (GCMS)

Urine samples were collected over a 24 -h period. Subsequently, $1.0 \mathrm{~mL}$ urine sample was transferred into polypropylene test tube, to which $1 \mathrm{~g}$ of $\mathrm{NaCl}, 200 \mu \mathrm{L}$ of $5 \mathrm{~N} \mathrm{HCl}$ and $6 \mathrm{~mL}$ of ethyl acetate were added. The mixture was shaken well for $10 \mathrm{~min}$ before being centrifuged at 2,236 $\mathrm{g}$ for $3 \mathrm{~min}$. The extracts were then prepared to be injected into the GCMS instrument.

\section{Measurement of lipid peroxidation products}

The levels of lipid peroxidation in the plasma and testes homogenate were measured as TBARS using an oxiSelect TBARS assay kit (MDA Quantitation, Cell Biolabs, CA, USA, cat. no. STA-330). Plasma samples were assayed directly using the kit. The testes were first homogenized on ice at $100 \mathrm{mg} / \mathrm{mL}$ in PBS containing $1 \times$ butylated hydroxytoluene $(\mathrm{BHT})$ provided in the kit, and were subsequently centrifuged at 10,000 $\mathrm{g}$ for $5 \mathrm{~min}$ to collect the supernatant. The TBARS assay was applied to $100 \mathrm{uL}$, while another $100 \mathrm{uL}$ was utilized for protein normalization (Bio-Rad Laboratories, CA, USA, cat. no. 500-0201EDU).

\section{Measurement of protein damage products}

Protein carbonyls were measured from cell homogenates using 2,4-dinitrophenylhydrazine derivatization according to the manufacturer's instructions (Protein Carbonyl Assay Kit, cat. no. ab126287; Abcam, Cambridge, MA).

Advanced oxidation protein product (AOPP) levels were analyzed by use of an OxiSelect AOPP kit (STA-318, Cell Biolabs, San Diego, CA, USA). Samples containing $250 \mu \mathrm{g} / \mathrm{mL}$ protein were prepared. Constant volumes of $200 \mu \mathrm{L}$ were added to the wells of a microtiter plate (Cell Biolabs) before being exposed to chloramine reaction initiator, followed by stop solution. Absorbance was recorded by use of a spectrophotometric plate reader, with $340 \mathrm{~nm}$ as the primary wavelength.

\section{Measurement of TAC}

TAC of serum was measured using the TAC kit (Abcam, Cam- bridge, UK) according to the manufacturer's instructions. Briefly, plasma was allowed to reduce $\mathrm{Cu}_{2}+$ for $1.5 \mathrm{~h}$ at room temperature. Reduced $\mathrm{Cu}+$ was chelated with a colorimetric probe and absorbance was measured at $570 \mathrm{~nm}$. Results were expressed as trolox equivalent, according to a trolox standard curve.

\section{Statistical analysis}

Statistical analysis was carried out using SPSS v.13.0 software. Non-parametric analysis was performed, as the data were not normally distributed (Kolmogorov-Smirnov test, $\mathrm{P}<0.05$ ). Means and standard errors were also calculated. Mann-Whitney $U$ test was performed to compare oxidative stress marker levels of the two groups. Spearman's correlation was used to determine the association between oxidative stress marker levels and patient's age, disease severity scores and HGA levels. Multiple linear regression models were adopted to test the relationship between serum protein carbonyl levels and the disease severity score in AKU patients.

The study protocol was approved by the Mutah University's Ethics Committee on human research.

\section{Results}

\section{Patients characteristics}

The study sample included 21 AKU patients (mean age $37.9 \pm$ 12.6 years, range 13 - 58, 17 male, 4 female) and 19 age- and sex-matched healthy controls (mean age $36 \pm 10.6$ years, range 13 - 53, 13 male, 6 female).

The AKU diagnosis was based on urinary HGA tests and clinical examination results. The HGA levels in controls were null. The clinical features of the disease in the patients are summarized in Table 1.

\section{Mean concentrations of the oxidation and anti-oxidation mediators in the serum of AKU patients and healthy con- trols}

Mean plasma levels and the standard error of patient's age, total antioxidant capacity, oxidative mediators TBARS, PC and AOPP in both the AKU and the control group, and the disease severity score and HGA urine levels in the AKU patients were measured and the findings are reported in Table 2. The plasma levels of TBARS, PC and TAC were found to be high in AKU patients but low in controls; whereas plasma level of AOPP was nearly the same in the two groups. The greatest mean value was noted for TAC (1.56-fold change with respect to the controls), followed by PC and TBARS (1-fold change with respect to the control group). Finally, similar AOPP levels were noted in both alkaptonuria patients and controls. The Mann-Whitney $\mathrm{U}$ test revealed a significant difference in oxidative markers TBARS and TAC in the AKU patients compared with the controls $(\mathrm{P}<0.05)$, as shown in Table 2 . Regarding the results of 
Table 1. Patient's Clinical Features

\begin{tabular}{ll}
\hline Clinical features/symptoms & N (\%) \\
\hline Dark urine & $21(100 \%)$ \\
Pigmentation of skin, outer ear and sclera of the eye & $16(76 \%)$ \\
Teeth pigmentation & $19(90 \%)$ \\
Morning stiffness and back pain due to degenerate arthropathy & $21(100 \%)$ \\
Arthropathy of large joints (hips and shoulders) & $21(100 \%)$ \\
Knees pain & $13(62 \%)$ \\
Kidney stones & $13(62 \%)$ \\
Prostate stones & $5(24 \%)$ \\
Aortic stenosis & $1(5 \%)$ \\
Ruptures of muscles, tendons and ligaments & $1(5 \%)$ \\
Knee joint replacement & $1(5 \%)$ \\
Foot pain and swelling of small joints (interphalangeal joints) & $1(5 \%)$ \\
\hline
\end{tabular}

Table 2. Age, Plasma Oxidative Markers' Levels, Disease Severity Score and Urine HGA Levels in Alkaptonuria Patients and Controls

\begin{tabular}{lllll}
\hline Variable & Patients $^{\mathbf{a}}$ & Control $^{\mathbf{a}}$ & Mann-Whitney U & P value \\
\hline Age (years) & $37.86 \pm 12.59$ & $36.00 \pm 10.62$ & $174.5 \mathrm{~A}$ & 0.496 \\
Sex $(\mathrm{F} / \mathrm{M})$ & $4 / 17$ & $6 / 13$ & & $0.00^{*}$ \\
TAC $(\mathrm{nmol} / \mu \mathrm{L}$ copper reducing equivalent) & $13.82 \pm 1.63$ & $8.92 \pm 1.63$ & 6 & $0.00^{*}$ \\
TAC $(\mathrm{nmol} / \mu \mathrm{L}$ uric acid equivalent) & $0.63 \pm 0.074$ & $0.41 \pm 0.074$ & 6 & $0.002^{*}$ \\
TBARS $(\mathrm{MDA})(\mu \mathrm{mol} / \mathrm{L})$ & $0.050 \pm 0.001$ & $0.049 \pm 0.0005$ & 84.5 & 0.076 \\
Protein carbonyl $(\mathrm{nmol} / \mathrm{mL})$ & $1.87 \pm 0.34$ & $1.77 \pm 0.23$ & 134 & 0.297 \\
AOPP $(\mu \mathrm{mol} / \mathrm{mL})$ & $4.39 \pm 0.11$ & $4.39 \pm 0.14$ & 161 & - \\
HGA level $(\mathrm{g} / \mathrm{day})$ & $1.23 \pm 0.98$ & - & - & - \\
Disease severity score & $34.24 \pm 22.25$ & - & & - \\
\hline
\end{tabular}

Patient score and urine HGA were null in controls. ${ }^{a}$ Data are means \pm standard deviation (SD). ${ }^{*} \mathrm{P}$ value $<0.05$ versus healthy subjects.

TBARS (MDA) among patients and controls; mathematically and statistically very small differences $(0.001)$ was detected as significant. These result were expected as the standard deviation and standard error were extremely very low with variance

Table 3. Correlation of the Patient's Age, Patients' Plasma TAC, TBARS, PC, AOPP and HGA Levels With Disease Severity Score in the AKU Patients

\begin{tabular}{llc}
\hline \multirow{2}{*}{ Variable } & \multicolumn{2}{c}{ Disease severity score } \\
\cline { 2 - 3 } & Spearman's correlation $(\mathbf{r})$ & P value \\
\hline Disease severity score & 1 & $0.000^{*}$ \\
Age (years) & 0.557 & 0.353 \\
TAC $(\mathrm{nmol} / \mu \mathrm{L}$ copper reducing equivalent) & -0.046 & 0.353 \\
TAC $(\mathrm{nmol} / \mu \mathrm{L}$ uric acid equivalent) & -0.046 & 0.673 \\
TBARS $(\mathrm{MDA})(\mu \mathrm{mol} / \mathrm{L})$ & -0.098 & $0.024 *$ \\
Protein carbonyl $(\mathrm{nmol} / \mathrm{mL}$ & 0.242 & 0.530 \\
AOPP $(\mu \mathrm{mol} / \mathrm{mL})$ & 0.021 & 0.494 \\
HGA level $(\mathrm{g} / \mathrm{day})$ & -0.043 & \\
\hline
\end{tabular}

${ }^{*} \mathrm{P}$ value $<0.05$ 

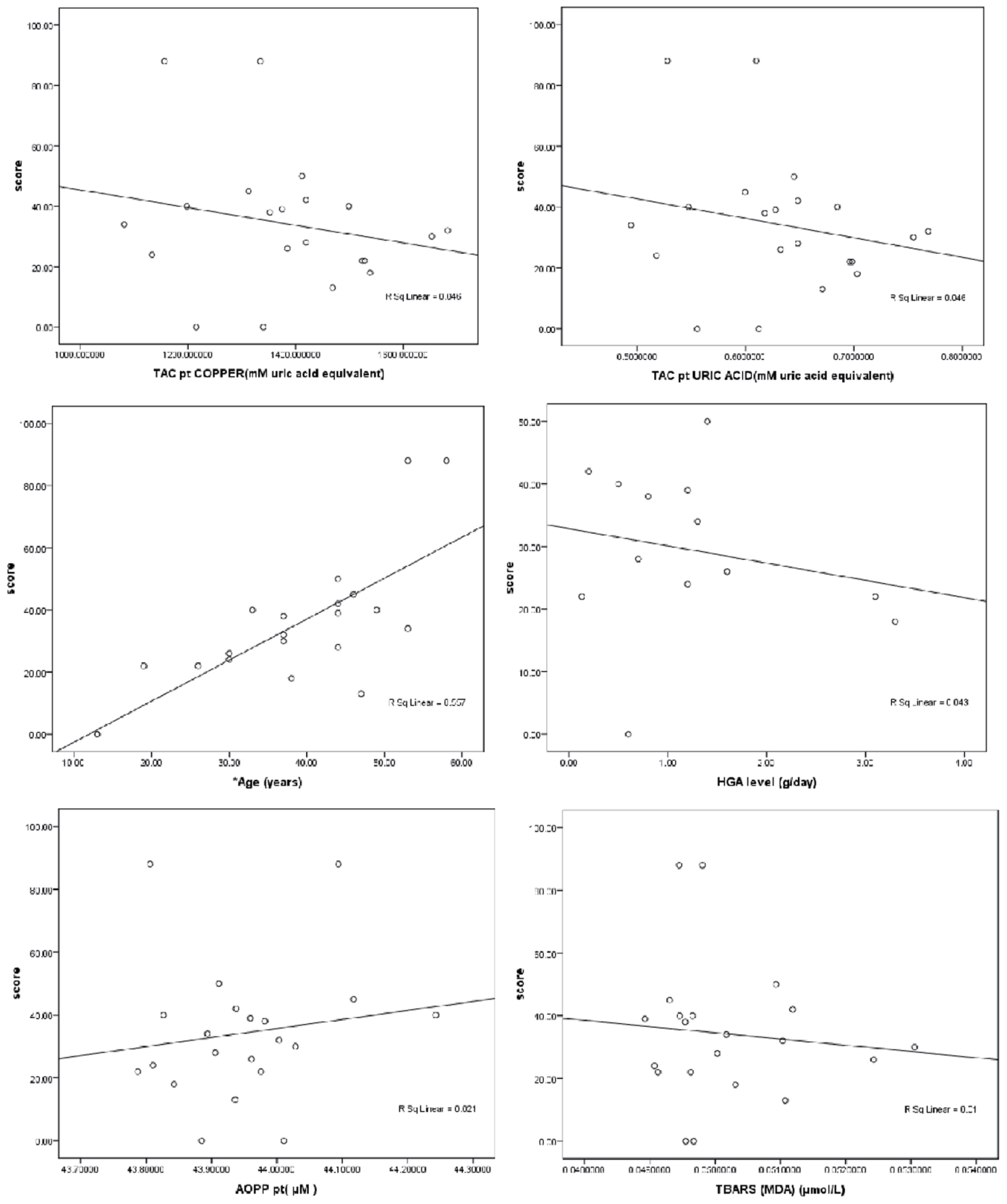

Figure 1. Scatter plot of the patient's age, patients' plasma TAC, TBARS, AOPP and HGA levels with disease severity score in the AKU patients.

approximate zero.

\section{Correlation of the oxidative markers, age and HGA with AKU severity score}

Using Spearman's correlation, each of the oxidative markers (TAC, TBARS, PC and AOPP) in patients' plasma and patient age were correlated with the disease severity score. The find- ings indicated that the disease severity score was positively correlated with the patients' $\mathrm{PC}$ and age $(\mathrm{r}=0.49$ and 0.75 , respectively; $\mathrm{P}<0.05$ ), as shown in Table 3 and Figure 1.

\section{The factors associated with the $\mathrm{AKU}$ disease}

After adjusting for the confounding factors (patient age, TAC, TBARS and AOPP), $\mathrm{PC}$ was shown to have a higher predictive 
Table 4. Linear Regression Analysis for Disease Severity Score Prediction

\begin{tabular}{|c|c|c|c|c|c|c|}
\hline \multirow{2}{*}{ Model } & \multicolumn{2}{|c|}{ Unstandardized coefficients } & \multirow{2}{*}{$\begin{array}{l}\text { Standardized coefficients } \\
\text { Beta }\end{array}$} & \multirow{2}{*}{ P value } & \multicolumn{2}{|c|}{$95 \%$ confidence interval for $B$} \\
\hline & B & SE & & & Lower bound & Upper bound \\
\hline Constant & 22.239 & 23.343 & & 0.353 & -71.1 & 26.620 \\
\hline
\end{tabular}

SE: standard error. *P value $<0.05$.

value for the disease severity score in the AKU patients. The following linear equation describes this relationship: disease severity score $=22.24+0.0 \times \mathrm{PC}$. The regression results reported in Table 4 and Figure 2 indicate that $\mathrm{PC}$ is an important oxidative factor affecting the disease.

\section{Discussion}

In the present study, patients diagnosed with AKU had a significantly higher extent of lipid damage (TBARS) and TAC levels compared to the healthy controls. Although the plasma PC levels were non-significantly higher in AKU patients, they seemed to correlate with and predict the disease severity score in AKU patients, as indicated by the equation given above and the findings presented in Table 4 and Figure 2. Thus, it can be concluded that PC is an important mediating factor in joint inflammation in AKU patients. The AOPP concentrations were similar between patients and controls.

The role of oxidation had been previously reported in AKU chondrocytes model. It has been postulated that oxidation of the HGA into HGD and production of ROS is the initiating event of ochronotic arthritis. Moreover, it was demonstrated that pigmented areas in AKU cartilage coincide with the areas of oxidation $[5-8,23]$. This is followed by the induc- tion of inflammation in the synovial membrane and the lining of the joints.

Protein oxidation and lipid peroxidation were discussed in previous studies on AKU. Authors of one study suggested that the morphological changes of chondrocytes in AKU cartilage may be attributed to lipid peroxidation generating large amounts of reactive products, which have been strongly implicated in the mechanisms of HGA-induced cartilage apoptosis [9]. Moreover, findings yielded by another study showed pathological levels of serum and plasma AOPP from alkaptonuric individuals [24]. Previous studies on oxidative stress in humans further showed that lipid peroxidation detected in AKU aortic valve was probably induced by HGA and its oxidized products. An evident co-localization between oxidized lipids, lymphocyte and macrophage accumulation, and ochronotic deposits suggests that lipid peroxidation might play a role in the disease process $[25,26]$.

It is proposed that protein carbonyl derivatives are generated by both direct oxidative attack of proteins and/or by indirect lipid peroxidation products, and therefore represent a good biomarker for general oxidative stress [27], which may explain why PC, rather than TBARS, is the only factor significantly associated with the disease severity score in the present study.

In patients diagnosed with alkaptonuria, aging is associated with progressive disability [28]. In the present study, this

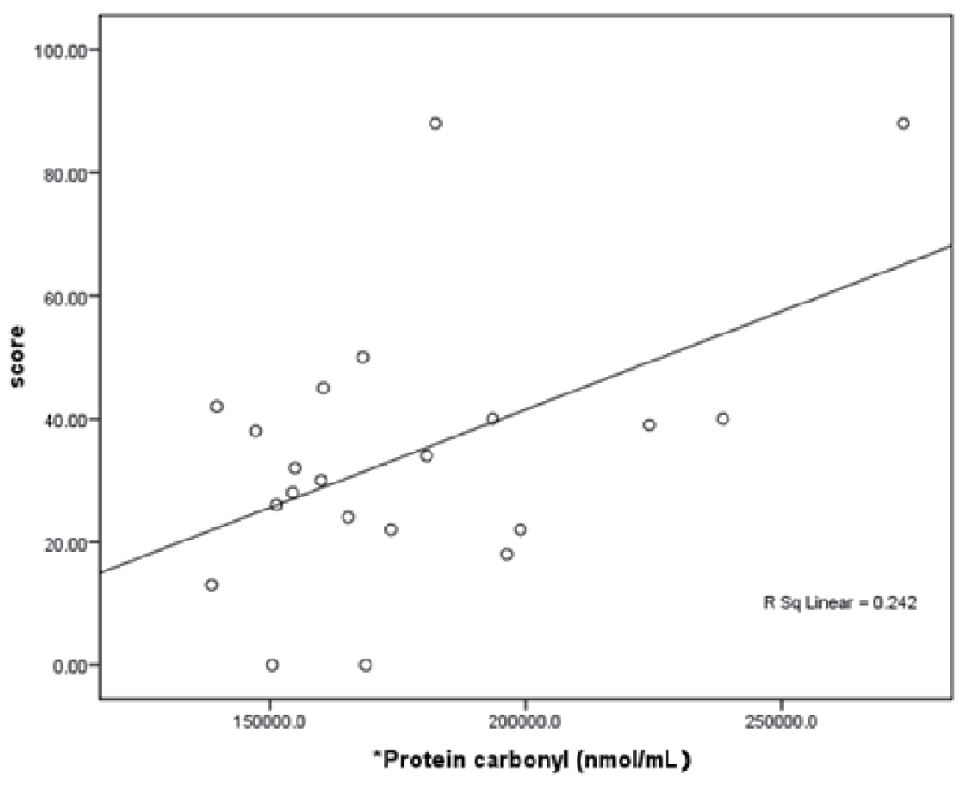

Figure 2. Scatter plot of protein carbonyl in AKU patients. 
link was confirmed by as a statistically significant correlation between patient's age and the disease score.

Finally, some limitations of our study should be recognized. First, a measure of other compounds of oxidant and enzymatic antioxidant states would be highly beneficial, as it would permit more accurate evaluation of this balance. Second, the lack of ROS determination in AKU samples prevented establishment of a meaningful relationship between ROS levels and oxidation state in patients.

Due to the rarity of the disease, the present study findings require further prospective validation using a mouse model studies and human studies with larger sample sizes. Such comprehensive evaluations would ensure that the complications associated with the disease can be prevented, or at least mitigated by controlling the oxidation levels in the patients.

Furthermore, the effect of antioxidant status needs to be studied further for the possible effects of dietary supplementations with sources of antioxidant vitamins and polyphenols on antioxidant capacity and oxidation [29-31].

\section{Conclusions}

Some of the HGA-induced oxidative stress in AKU patients was discussed in this paper, such as an increasing oxidative damage of protein and lipids. The reported findings related to increased anti-oxidant capacity are attributed to compensated increased oxidation molecules in AKU patients. These changes may help in the clarification of the molecular mechanisms of ochronosis. Moreover, having the predictive model for alkaptonuria disease severity scoring that combines PC levels with clinical data would be extremely useful in the assessment of organ dysfunction, in monitoring disease progression, and potentially in providing the basis for its pharmacological treatment.

\section{Acknowledgments}

The research team is thankful to all school directors and teachers who helped us in data collection.

\section{Conflict of Interest}

The authors declare no conflict of interest.

\section{Informed Consent}

Written informed consent was obtained from all of the patients who participated. All of the researchers conducted the study in accordance with the Declaration of Helsinki.

\section{Financial Disclosure or Funding}

Not applicable.

\section{Author Contributions}

All authors participated to review, and were involved in writing and revising the article prior to submission.

\section{References}

1. Sampson HA, Burks A. Adverse reactions to foods. Allergy: Principles and Practice (Middleton E, Reed CE, Elliset EF, eds), 4th ed. St Louis: CV Mosby Co. 1993;16611686.

2. Hong X, Tsai HJ, Wang X. Genetics of food allergy. Curr Opin Pediatr. 2009;21(6):770-776.

3. Di Giovine FS, Malawista SE, Nuki G, Duff GW. Interleukin 1 (IL 1) as a mediator of crystal arthritis. Stimulation of $\mathrm{T}$ cell and synovial fibroblast mitogenesis by urate crystal-induced IL 1. J Immunol. 1987;138(10):32133218.

4. Levy MB, Goldberg MR, Nachshon L, Tabachnik E, Katz Y. Lessons from cases of mortality due to food allergy in Israel: cow's milk protein should be considered a potentially fatal allergen. Isr Med Assoc J. 2012;14(1):29-33.

5. Dinarello CA. Blocking interleukin-1beta in acute and chronic autoinflammatory diseases. J Intern Med. 2011;269(1):16-28.

6. Tanaka Y. [Pathological mechanisms in rheumatoid arthritis]. Nihon Rinsho. 2013;71(7):1147-1152.

7. Tilg H, Trehu E, Atkins MB, Dinarello CA, Mier JW. Interleukin-6 (IL-6) as an anti-inflammatory cytokine: induction of circulating IL-1 receptor antagonist and soluble tumor necrosis factor receptor p55. Blood. 1994;83(1):113-118.

8. Ho MH, Lee SL, Wong WH, Ip P, Lau YL. Prevalence of self-reported food allergy in Hong Kong children and teens - a population survey. Asian Pac J Allergy Immunol. 2012;30(4):275-284.

9. Benyamini Y, Leventhal EA, Leventhal H. Gender differences in processing information for making self-assessments of health. Psychosom Med. 2000;62(3):354-364.

10. Iqbal I, Fleischmann R. Treatment of osteoarthritis with anakinra. Curr Rheumatol Rep. 2007;9(1):31-35.

11. Perry MB, Suwannarat P, Furst GP, Gahl WA, Gerber LH. Musculoskeletal findings and disability in alkaptonuria. J Rheumatol. 2006;33(11):2280-2285.

12. Feldmann M, Brennan FM, Maini RN. Role of cytokines in rheumatoid arthritis. Annu Rev Immunol. 1996;14:397440.

13. Kjellman NI. Atopic disease in seven-year-old children. Incidence in relation to family history. Acta Paediatr Scand. 1977;66(4):465-471.

14. Branum AM, Lukacs SL. Food allergy among U.S. children: trends in prevalence and hospitalizations. NCHS Data Brief. 2008;10:1-8.

15. van Odijk J, Kull I, Borres MP, Brandtzaeg P, Edberg U, Hanson LA, Host A, et al. Breastfeeding and allergic disease: a multidisciplinary review of the literature (1966-2001) on the mode of early feeding in infancy 
and its impact on later atopic manifestations. Allergy. 2003;58(9):833-843.

16. Ranganath LR, Cox TF. Natural history of alkaptonuria revisited: analyses based on scoring systems. J Inherit Metab Dis. 2011;34(6):1141-1151.

17. Keller JM, Macaulay W, Nercessian OA, Jaffe IA. New developments in ochronosis: review of the literature. Rheumatol Int. 2005;25(2):81-85.

18. Martin JP, Jr., Batkoff B. Homogentisic acid autoxidation and oxygen radical generation: implications for the etiology of alkaptonuric arthritis. Free Radic Biol Med. 1987;3(4):241-250.

19. Mateen S, Moin S, Khan AQ, Zafar A, Fatima N. Increased reactive oxygen species formation and oxidative stress in rheumatoid arthritis. PLoS One. 2016;11(4):e0152925.

20. Majkowska-Wojciechowska B, Wardzynska A, Luczynska M, Kowalski M, Makowska J, Kowalski M. Food hypersensitivity in the population of school children in Lodz-results of the "EuroPrevall" surveys. Alergia Astma Immunologia. 2009;14(1):35-44.

21. Kagan RS. Food allergy: an overview. Environ Health Perspect. 2003;111(2):223-225.

22. Uguz A, Lack G, Pumphrey R, Ewan P, Warner J, Dick J, Briggs D, et al. Allergic reactions in the community: a questionnaire survey of members of the anaphylaxis campaign. Clin Exp Allergy. 2005;35(6):746-750.

23. Webb LM, Lieberman P. Anaphylaxis: a review of 601 cases. Ann Allergy Asthma Immunol. 2006;97(1):39-43.

24. Sicherer SH, Sampson HA. 9. Food allergy. J Allergy Clin
Immunol. 2006;117(2 Suppl Mini-Primer):S470-475.

25. Phornphutkul $\mathrm{C}$, Introne WJ, Perry MB, Bernardini I, Murphey MD, Fitzpatrick DL, Anderson PD, et al. Natural history of alkaptonuria. N Engl J Med. 2002;347(26):2111-2121.

26. Gaines JJ, Jr., Tom GD, Khankhanian N. An ultrastructural and light microscopic study of the synovium in ochronotic arthropathy. Hum Pathol. 1987;18(11):1160-1164.

27. Koletzko S, Niggemann B, Arato A, Dias JA, Heuschkel R, Husby S, Mearin ML, et al. Diagnostic approach and management of cow's-milk protein allergy in infants and children: ESPGHAN GI Committee practical guidelines. J Pediatr Gastroenterol Nutr. 2012;55(2):221-229.

28. Burr ML, Merrett TG. Food intolerance: a community survey. Br J Nutr. 1983;49(2):217-219.

29. Castilla P, Echarri R, Davalos A, Cerrato F, Ortega H, Teruel JL, Lucas MF, et al. Concentrated red grape juice exerts antioxidant, hypolipidemic, and antiinflammatory effects in both hemodialysis patients and healthy subjects. Am J Clin Nutr. 2006;84(1):252-262.

30. Basu A, Betts NM, Ortiz J, Simmons B, Wu M, Lyons TJ. Low-energy cranberry juice decreases lipid oxidation and increases plasma antioxidant capacity in women with metabolic syndrome. Nutr Res. 2011;31(3):190-196.

31. Winklhofer-Roob BM, Rock E, Ribalta J, Shmerling DH, Roob JM. Effects of vitamin E and carotenoid status on oxidative stress in health and disease. Evidence obtained from human intervention studies. Mol Aspects Med. 2003;24(6):391-402. 